\title{
Comparative effectiveness and cost- effectiveness of Chuna manual therapy versus conventional usual care for nonacute low back pain: study protocol for a pilot multicenter, pragmatic randomized controlled trial (pCRN study)
}

Byung-Cheul Shin ${ }^{1,2}$, Me-riong Kim³ ${ }^{3}$ Jae-Heung Cho ${ }^{4}$, Jae-Young Jung ${ }^{4}$, Koh-Woon Kim, Jun-Hwan Lee,6, Kibong Nam${ }^{7}$, Min ho Lee ${ }^{7}$, Eui-Hyoung Hwang ${ }^{1,2}$, Kwang-Ho Heo ${ }^{1,2}$, Namkwen Kim $^{8}$ and In-Hyuk Ha ${ }^{3 *}$ (D)

\begin{abstract}
Background: While Chuna manual therapy is a Korean manual therapy widely used primarily for low back pain (LBP)-related disorders in Korea, well-designed studies on the comparative effectiveness of Chuna manual therapy are scarce.
\end{abstract}

Methods/design: This study is the protocol for a three-armed, multicenter, pragmatic randomized controlled pilot trial. Sixty severe nonacute LBP patients (pain duration of at least 3 weeks, Numeric Rating Scale (NRS) $\geq 5$ ) will be recruited at four Korean medicine hospitals. Participants will be randomly allocated to the Chuna group $(n=20)$, usual care group $(n=20)$, or Chuna plus usual care group $(n=20)$ for 6 weeks of treatment. Usual care will consist of orally administered conventional medicine, physical therapy, and back pain care education. The trial will be conducted with outcome assessor and statistician blinding. The primary endpoint will be NRS of LBP at week 7 post randomization. Secondary outcomes include NRS of leg pain, the Oswestry Disability Index (ODI), the Patient Global Impression of Change (PGIC), the Credibility and Expectancy Questionnaire, lumbar range of motion (ROM), the EuroQol-5 Dimension (EQ-5D) health survey, the Health Utility Index III (HUI-III), and economic evaluation and safety data. Post-treatment follow-ups will be conducted at 1, 4, and 10 weeks after conclusion of treatment.

Discussion: This study will assess the comparative effectiveness of Chuna manual therapy compared to conventional usual care. Costs and effectiveness (utility) data will be analyzed for exploratory cost-effectiveness analysis. If this pilot study does not reach a definite conclusion due to its small sample size, these results will be used as preliminary results to calculate sample size for future large-scale clinical trials and contribute in the assessment of feasibility of a full-scale multicenter trial.

Trial registration: Clinical Research Information Service (CRIS), KCT0001850. Registered on 17 March 2016. Keywords: Low back pain, Spinal manipulation, Cost-benefit analysis, Complementary therapies, Protocol

\footnotetext{
*Correspondence: hanihata@gmail.com

${ }^{3}$ Jaseng Spine and Joint Research Institute, Jaseng Medical Foundation, 858

Eonju-ro, Gangnam-gu, Seoul 135-896, Republic of Korea

Full list of author information is available at the end of the article
} 


\section{Background}

\section{Background and rationale}

Low back pain (LBP) is a highly prevalent musculoskeletal disorder that incurs severe pain, increase in sick leave and substantial social costs [1], is disabling to both individual and society and affects approximately 70-80\% adults over their lifetimes [2]. Therefore, appropriate LBP treatment selection is of considerable importance for individual patients, physicians, and decision-makers of health care policy.

Spinal manipulative therapy (SMT) is extensively used for acute and chronic LBP, and its efficacy has been reported in numerous randomized controlled trials (RCTs) and systematic reviews (SRs). However, upon closer inspection, the two Cochrane reviews on SMT for acute and chronic LBP mainly use search terms such as manipulation, osteopathy, and chiropractic, and do not include Korean Chuna manual therapy or Chinese Tuina [3]. Moreover, it can be reasonably inferred that though SMT is widely recommended for both acute and chronic LBP in various guidelines [4-6], the vast disparity in background history, diagnosis and treatment principles and techniques precludes unitary appreciation of treatment effect.

Chuna, literally meaning manual treatment in Korean, is a Korean spinal manipulation that has developed absorbing aspects of Chinese Tuina, chiropractic manipulation, and osteopathic medicine over time. Manipulative therapy can, therefore, be likened to a reservoir rich with academic contributions from various health care disciplines, drawing from, and emptying into, reserves to form a continuous cycle. Chuna incorporates radiology-based diagnosis, which is not customary for Chinese SMT, and includes meridian theory, meridian muscle concepts, and organ pattern identification diagnosis not usually seen in chiropractic manipulation or osteopathic medicine [7]. However, a recent study assessing the Chuna literature published from 1995 to May 2013 in Korea reported that clinical studies mainly consisted of case series $(n=99 ; 72 \%)$, RCTs $(n=15$; $11 \%)$, and non-RCTs $(n=21 ; 16 \%)$ [8]. While the proportion of RCTs in Chuna-related studies is steadily increasing, most studies take on an integrative approach as opposed to single treatment, and are not well-equipped to rigorously evaluate its therapeutic effect. Also, in quality assessment of RCTs in accordance with the Consolidated Standards of Reporting Trials (CONSORT) Statement [9], weaknesses regarding identification in title or structured summary in abstract, inadequate trial design description, statistical limitation from small sample size, inaccuracy of randomization and allocation process, difficulty in blinding, and insufficient reporting of participant flow and estimated effect size of interventions and its precision were reported [10].
While Chuna manual therapy is primarily used for LBP in Korea with high patient satisfaction [11], it is currently not covered by national health insurance and thus accessibility due to cost is limited considering its high satisfaction rate [12]. The Korean Ministry of Health and Welfare's 2011 report on usage of Korean medicine shows that Chuna was one of the most common usages of Korean medicine [11]. At the 2011 National Assembly forum for insurance guarantee reinforcement of Korean medicine, increasing national insurance coverage of Korean medicine treatments, such as Chuna manual therapy, was proposed with the aim of increasing accessibility to Korean medicine services, promoting national health, and improving quality of life [13]. Therefore, well-designed, high-quality RCTs are highly needed in the research sector to determine the effectiveness and economic value of Chuna manual therapy and to meet social demand.

\section{Objectives}

Objective evaluation of the comparative effectiveness, safety, and economic evaluation of Chuna manual therapy for application in cost-effective, evidencebased practice are important issues both for the Korean medicine sector and for national health services improvement. High-quality RCTs are warranted for rigorous assessment of Chuna effectiveness, safety, and cost-effectiveness. The hypotheses of this study are as follows: (1) Chuna manual therapy will be more effective and safer than (or equally safe as) conventional usual care in nonacute LBP patients and (2) concurrent treatment consisting of Chuna manual therapy and usual care will be more effective than Chuna alone or conventional usual care alone in nonacute LBP patients. Therefore, the objectives of this pCRN (pilot Chuna Research Network) study are to investigate the following in nonacute LBP patients: (1) the comparative effectiveness and safety of Chuna manual therapy compared to conventional usual care through comparison of pain, functionality, quality of life parameters and adverse events, (2) the effectiveness and safety of a concurrent treatment consisting of Chuna manual therapy and usual care, which is reflective of actual clinical practice, through comparison of two single treatment groups of Chuna manual therapy and usual care, and a combined treatment group of Chuna manual therapy with usual care and (3) costs and effectiveness (utility) data of the three groups for exploratory cost-effectiveness analysis (economic evaluation). However, if the current sample size deters this pilot study from reaching a definite conclusion regarding aims (1), (2), and (3), the main objectives of this preliminary study will be to determine a sample size appropriate for statistical testing 
in future large-scale studies and to assess the feasibility of a full-scale multicenter trial.

\section{Methods/design}

The protocol of this pCRN study is presented in accordance with the 2013 SPIRIT (Standard Protocol Items: Recommendations for Interventional Trials) Statement (see Additional file 1 for the populated SPIRIT Checklist and Table 1 for the trial schedule of enrollment, interventions, and assessments in accordance with recommended SPIRIT figure).

\section{Trial design and study setting}

This pCRN study employs a multicenter, randomized, three-armed, parallel-group design. Participants will be randomly allocated to the Chuna manual therapy group, a usual care group, or a combined treatment group of Chuna manual therapy with usual care at a ratio of 1:1:1. Participants will be recruited at four major Korean medicine hospitals in Korea (Pusan National University Korean Medicine Hospital, Kyung Hee University Korean Medicine Hospital at Gangdong, Jaseng Hospital of Korean Medicine, and Mokhuri Neck and Back Hospital) and recruitment period is anticipated to be from February to August 2016. Participants will be recruited through each hospital website and online and offline advertisements. These study sites possess the facilities, personnel, and patient recognition required for LBP patient recruitment, and further information on the study sites is available at Clinical Research Information Service (CRIS registration number: KCT0001850). Participants will receive a total 6 weeks of outpatient treatment for LBP starting from visit 2 through random allocation if deemed eligible in screening at visit 1 . General management and treatment processes will adhere to predetermined clinical trial procedures.

The entire research process, design, and setting were prepared through six or more meetings of 22 pCRN

Table 1 Time points of each assessment index

\begin{tabular}{|c|c|c|c|c|c|c|c|c|c|c|c|}
\hline \multirow{3}{*}{ Time point } & \multicolumn{11}{|c|}{ Study period } \\
\hline & \multirow{2}{*}{$\begin{array}{l}\text { Enrollment } \\
\text { Week -1 }\end{array}$} & \multirow{2}{*}{$\begin{array}{l}\text { Allocation } \\
\text { Week } 0 \text { (Baseline) }\end{array}$} & \multicolumn{6}{|c|}{ Active treatment post allocation } & \multicolumn{3}{|c|}{ Follow-up } \\
\hline & & & $\begin{array}{l}\text { Week } \\
1\end{array}$ & $\begin{array}{l}\text { Week } \\
2\end{array}$ & $\begin{array}{l}\text { Week } \\
3\end{array}$ & $\begin{array}{l}\text { Week } \\
4\end{array}$ & Week 5 & Week 6 & $\begin{array}{l}\text { Week } \\
7\end{array}$ & $\begin{array}{l}\text { Week } \\
10\end{array}$ & $\begin{array}{l}\text { Week } \\
14\end{array}$ \\
\hline \multicolumn{12}{|l|}{ Enrollment } \\
\hline Eligibility screening & $\circ$ & & & & & & & & & & \\
\hline Written informed consent & $\circ$ & & & & & & & & & & \\
\hline Vital signs & $\circ$ & & & & & & & & $\circ$ & $\circ$ & $\circ$ \\
\hline $\begin{array}{l}\text { Sociodemographic characteristics, } \\
\text { medical history (e.g., LBP, medication } \\
\text { history) }\end{array}$ & $\circ$ & & & & & & & & & & \\
\hline Physical examination & $\circ$ & & & & & & & & & & \\
\hline Randomized allocation & & $\circ$ & & & & & & & & & \\
\hline \multicolumn{12}{|l|}{ Interventions } \\
\hline Treatment in CMT group & & & $\leftarrow 2-3$ & times/v & veek $\rightarrow$ & & $\leftarrow 1-3$ tir & es/week $\rightarrow$ & & & \\
\hline Treatment in $U C^{a}$ group (times/week) & & & $\leftarrow 2-3$ & times/V & reek $\rightarrow$ & & $\leftarrow 1-3$ tir & es/week $\rightarrow$ & & & \\
\hline $\begin{array}{l}\text { Treatment in CMT }+U C^{\mathrm{a}} \text { group } \\
\text { (times/week) }\end{array}$ & & & $\leftarrow 2-3$ & times/v & reek $\rightarrow$ & & $\leftarrow 1-3$ tir & es/week $\rightarrow$ & & & \\
\hline \multicolumn{12}{|l|}{ Assessments } \\
\hline Symptoms and change in medicine & & $\circ$ & & $\circ$ & $\circ$ & $\circ$ & $\circ$ & $\circ$ & $\circ$ & $\circ$ & \\
\hline NRS of LBP and leg pain & $\circ$ & $\circ$ & & $\circ$ & o & $\circ$ & $\circ$ & $\circ$ & $\circ$ & $\circ$ & $\circ$ \\
\hline ODI & & $\circ$ & & & & & $\circ$ & & $\circ$ & $\circ$ & \\
\hline PGIC & & & & & & & $\circ$ & & $\circ$ & $\circ$ & \\
\hline ROM & & $\circ$ & & & & & $\circ$ & & $\circ$ & $\circ$ & \\
\hline EQ-5D & & $\circ$ & & & & & $\circ$ & & $\circ$ & $\circ$ & $\circ$ \\
\hline HUI-III & & $\circ$ & & & & & $\circ$ & & $\circ$ & $\circ$ & $\circ$ \\
\hline Economic evaluation & & $\circ$ & & & & & $\circ$ & & $\circ$ & $\circ$ & $\circ$ \\
\hline Adverse events & & $\circ$ & $\leftarrow$ eve & ry visit & & & & & ० & $\circ$ & $\circ$ \\
\hline
\end{tabular}

CMT Chuna manual therapy, UC usual care, NRS Numeric Rating Scale, LBP low back pain, ODI Oswestry Disability Index, PGIC Patient Global Impression of Change, ROM range of movement, EQ-5D EuroQol-5 dimensions, HUI-III Health Utility Index III

aUC: medication will be administered daily and physical therapy will be applied 2-3 times/week 
members including rehabilitation experts $(n=14)$, clinical research coordinators $(n=4)$, clinical research methodologists $(n=2)$, and economic evaluation experts $(n=2)$ currently employed at six university hospitals, two spine-specialty Korean medicine hospitals, and the Korea Institute of Oriental Medicine (see Additional file 2 for trial committee organization and contributions and role).

\section{Participants}

\section{Inclusion criteria}

1. Nonacute LBP participants (with pain duration of 3 weeks or longer) requiring medical attention

2. Participants with average Numeric Rating Scale (NRS) $\geq 5$ during the past week

3. Participants aged 19 years or older, and 70 years or younger

4. Participants who have agreed to trial participation and provided written informed consent

\section{Exclusion criteria}

1. Participants diagnosed with serious pathology(s) which may cause LBP (e.g., spinal metastasis of tumor(s), acute fracture, spinal dislocation)

2. Participants with spinal surgery history within the last 3 months

3. Participants with other chronic disease(s) which may interfere with treatment effect or outcome interpretation (e.g., chronic renal failure)

4. Participants with progressive neurologic deficit or severe neurologic symptoms

5. Patients with an inner fixation or stabilization device mounted through spinal surgery

6. Participants currently taking steroids, immunosuppressants, medicine for psychological problems or other medication(s) that may interfere with study results

7. Participants who have received Chuna manual therapy or medicine which may influence pain levels, such as nonsteroidal anti-inflammatory drugs (NSAIDs), within the past week

8. Pregnant participants or participants who are planning to become pregnant

9. Subjects participating in other clinical studies or who are otherwise deemed unsuitable by the researchers

\section{Randomization and allocation concealment}

A statistician blinded to the objectives and exact design of this study will use SAS 9.3 to generate the allocation sequence using block randomization (block size $=6$ ). Researchers will take care so that block sizes do not become known to the researchers who conduct participant recruitment or intervention allocation.

The researcher conducting participant recruitment at each site (Korean medicine doctor (KMD)) will contact the main trial site for central randomization by phone for random allocation to the three groups at a ratio of 1:1:1 of participants who have voluntarily agreed to participate in the trial, given written informed consent, and been screened and found eligible for trial participation in accordance with inclusion and exclusion criteria. Central randomization by phone will be performed through contact upon participant enrollment by the researcher conducting participant recruitment at each site to a designated independent researcher at the main trial site who will consecutively assign the randomized number and allocation group of each participant by verifying the sequence in the order of phone contact. All allocation numbers will be concealed through blinding of randomized number generation and central phone randomization.

The four sites will receive random allocation competitively on participant enrollment without stratification by site, and each site will respectively stop recruitment when the equal allocated number of 15 participants has been recruited.

\section{Blinding}

As blinding of physicians and participants to allocation of treatment groups is impossible in the current design due to dissimilarity of interventions, only outcome assessors, statistician and data analysts will be blinded. The outcome assessors will not participate in treatment and a research nurse or KMD blinded to allocation will conduct outcome assessment in a separate room after treatment and will not attempt to become knowledgeable of allocation. The patient will also be cautioned against informing the outcome assessor of treatment allocation prior to each assessment. The electronic data transferred to statistician and data analysts will not contain information on treatment allocation.

\section{Interventions \\ Chuna manual therapy (see Additional file 3 for technical details with photos)}

A semistandardized Chuna treatment plan for LBP was established by technique selection based on specialist opinion collected through survey of Chuna clinical specialists (total $n=20$ ) [14] and physicians employed at a spine-specialty designated Korean medicine hospital [15]. The physicians administering Chuna in this trial will be KMDs with $3+$ years' clinical experience of Chuna manual therapy use, and will be deployed after receiving Chuna protocol training sessions (two sessions, $4+\mathrm{h} /$ session) for standardized application. Chuna covers a wide variety of techniques including high-velocity, 
low-amplitude thrusts to joints, slightly further than the passive range of motion, and mobilization which applies manual force to joints in the passive range of motion [16]. The Chuna techniques utilized in this study are divided into lumbar and pelvic regions, and mandatory or selective techniques will be performed following physician judgement (Table 2).

A total of 10-18 Chuna manual therapy sessions (at least 10 sessions or more are required to be received by participants) will be administered over a 6-week period. Frequency of treatment will differ by period with two to three sessions/week from week 1 to week 4 , and one to three sessions/week from week 5 to week 6 based on Chuna physician judgement of previous treatment results. The time duration of one Chuna manual therapy session will be approximately 15 min including evaluation and treatment.

\section{Usual care}

Conventional usual care will be limited to orally administered medicine, physical therapy, and back pain care education (15-min education with visual aids covering cause, prevention, management, and exercise for back pain care) in this study. Medication and physical therapy of usual care will be provided with reference to most common treatments used in LBP-related patients as assessed from 2011 Korean Health Insurance Review and Assessment (HIRA) statistics [17] or clinical practice guidelines, provided as lists of most frequent medicine and physical treatments to the usual care physician [5]. Participants will be asked to record drug intake to monitor adherence, and medicine and physical therapy usage type and frequency will be recorded in a separate Case Report Form (CRF) for outcome assessor blinding purposes.

\section{Combined Chuna manual therapy and usual care}

The combined treatment group of Chuna manual therapy and usual care will receive both treatments in the same method, frequency, session length, total duration, and number of sessions of each treatment as that of the other two groups.

\section{Cointerventions}

Additional treatment (e.g., other medication related to pain, acupuncture, procedures or surgery) other than that specified in the protocol will not be allowed during the 6-week intervention period.

\section{Outcomes \\ Primary outcome measurement}

The primary outcome will be LBP level for the past week assessed using the NRS. In the NRS, the participant selects a number from 0 to 10 that best indicates current pain level (with 0 indicating no pain, and 10 the most severe pain one can imagine) $[18,19]$.

\section{Secondary outcome measures}

Secondary outcome measures include leg pain level for the past week as assessed using the NRS. Functional status will be evaluated using the Oswestry Disability Index (ODI) questionnaire. The ODI is a 10-item questionnaire developed for the purpose of LBP-related disability assessment [20]. Each item has a six-level answer choice, and each level correlates with a score of 0-5. Higher scores reflect greater disability. The accredited Korean version of the ODI [21] will be used. The Patient Global Impression of Change (PGIC) will be assessed for comprehensive and global assessment of change in LBP and movement limitation due to pain $[18,22]$. The PGIC discerns subjective impression of change in seven levels ranging from $1=$ very much improved; $2=$ much improved; $3=$ minimally improved; $4=$ no change; $5=$ minimally worse; $6=$ much worse; to $7=$ very much worse. While the PGIC was initially developed for psychological use, it is currently applied in pain evaluation of various medical fields. The EuroQol-5 dimensions (EQ-5D) health survey is a measurement tool for health-related quality of life and widely used across the health care

Table 2 Semistandardized treatment plan of study Chuna techniques [38-41]

\begin{tabular}{|c|c|c|}
\hline Region & Mandatory techniques & Selective techniques \\
\hline Lumbar spine $^{a}$ & $\begin{array}{l}\text { - Spine flexion distraction technique (flexion) } \\
\text { - Sidelying lumbar (extension or flexion) dysfunction } \\
\text { correction technique }\end{array}$ & $\begin{array}{l}\text { - Spinal flexion distraction (circumduction) } \\
\text { - Spinal flexion distraction (sidebending) } \\
\text { - Iliopsoas fascial Chuna }\end{array}$ \\
\hline Pelvis ${ }^{a}$ & $\begin{array}{l}\text { <lliac dysfunction (if applicable by evaluation) > } \\
\text { - Prone iliac anterior rotation dysfunction correction } \\
\text { technique/ or } \\
\text { - Prone iliac posterior rotation/sacral sidebending } \\
\text { dysfunction correction technique } \\
\text { - Prone leg raise iliac dysfunction correction technique } \\
\text { - Prone inflare-outflare dysfunction correction technique } \\
\text { < Sacral dysfunction (if applicable by evaluation) > } \\
\text { - Prone sacral sidebending and rotation dysfunction } \\
\text { correction technique }\end{array}$ & $\begin{array}{l}\text { - Prone sacral dysfunction correction technique } \\
\text { (extension or flexion) }\end{array}$ \\
\hline
\end{tabular}

Dysfunction type assessed and diagnosed prior to treatment 
sector. The range of scores is from -1 , indicating health considered worse than death to 1 , meaning perfect health [23]. The EQ-5D comprises five dimensions or items assessing current health state, which are mobility, self-care, usual activities, pain/discomfort, and anxiety/ depression and graded into three levels (level $1=$ no problem; level 2 =some/moderate problem; and level 3 = extreme problem). This study will use a Korean estimate weighted-value model of health-related quality of life [24]

The Health Utility Index III (HUI-III) consists of health measures on sight, hearing, speaking, walking, agility, emotion, cognition, and pain, and quality of life values will be calculated by assigning section results to preference score calculation function following the method suggested by Kopec et al. [25].

Lumbar range of movement (ROM) will also be used to objectively assess improvement after treatment. While ROM evaluation is reliable $(r=0.94)$ and valid $(r=0.97)$ [26], it is not highly responsive (effect size 0.1-0.6) [27]. The angle between a perpendicular line and the patient's lumbar spine will be measured using a goniometer at maximum lumbar flexion, extension, sidebending, and rotation, respectively. If measurement is not possible due to pain, the angle will be recorded as $0^{\circ}$.

\section{Cost data investigation}

Costs associated with health care may be largely divided into three categories: medical, nonmedical, and lost productivity cost [28-30]. Medical cost covers direct expenses incurred by the use of medical facilities and services (official medical cost) and purchase of health foods and medical devices (unofficial medical cost). Nonmedical cost refers to accompanying costs incidental to medical use such as transportation, patient time, and nursing costs. Lost productivity cost represents economic loss from decreased work capability because of the disease itself or premature death due to disease. Cost data for economic evaluation will be collected through structured survey of medical, nonmedical, and lost productivity cost categories (basic items will be included or excluded according to period).

\section{Credibility and expectancy questionnaire}

The credibility and expectancy questionnaire will be used to assess treatment expectation on a 9-point Likert scale [31]. Participants will be asked to select an answer to the following questions on their first visit $(1=$ "not at all"; and 5="somewhat"; to 9= "very much"): "How much do you expect that Chuna manual therapy, usual care and combined treatment of Chuna with usual care will respectively alleviate your symptoms?"

\section{Sample size}

Determination of study sample size for pCRN: the following assumptions were made for calculation of valid sample size based on previous literature: (1) level of significance $(\alpha)=0.05,(2)$ type 2 error $(\beta)=0.2$; with test power set at $80 \%$ and (3) referring to NRS difference from prior Korean medicine research using the NRS as the primary measure [32,33], and average difference and standard deviation (SD) between the three groups specified as 1.5 and 2.5 , respectively, by a designated statistician, (4) compliance was set as $80 \%$.

G*Power 3.1.7 was used for sample size calculation, and based on (3) and applied values of mean $\pm \mathrm{SD}$, the derived effect size was 0.49. Consequent sample size, as determined by (1) and (2), and applying acquired sample size, was 45 (15 per group).

These results imply that to test the above hypotheses and factoring in a dropout rate of $25 \%$, each group requires a minimum $15 \times 100 / 75 \fallingdotseq 20$ (subjects), resulting in a total 60 participants.

\section{Statistical analysis}

Intention-to-treat (ITT) analysis will be the primary analysis used for all subjects who receive one or more treatment sessions, and additional per-protocol analysis will be performed in subjects who complete clinical trial participation, excluding dropouts. The primary endpoint will be at 1 week following the end of treatment (7 weeks post random allocation). Continuous data will be analyzed with a paired $t$ test for pre-post within-group difference and with an independent $t$ test for group comparison between Chuna and usual care if normally distributed, and the Wilcoxon rank sum test if not. For comparing between the three groups of Chuna with usual care, Chuna, and usual care, if continuous data satisfies the normality test, analysis of variance (ANOVA) will be used to test between-group difference, and the Kruskal-Wallis test if it does not. Chuna with usual care versus Chuna, and Chuna with usual care versus usual care will be analyzed using Duncan's post-hoc test. Post-hoc analysis of nonnormally distributed data will use the Wilcoxon rank sum test with significance level adjusted by the Bonferroni test. Categorical data will be analyzed using the chi-square test or Fisher's exact test. A paired $t$ test will be used in prepost within-group differences by time point. Secondary analyses will include analysis of covariance (ANCOVA) adjusting for statistically significant between-group differences in baseline outcomes or covariant factors at baseline, and subgroup analyses by LBP disease differentiation (nonspecific LBP, lumbar intervertebral disc herniation, and spinal stenosis), pain duration (subacute, chronic, and hyperchronic), and main site of pain (LBP worse than leg pain, and LBP less than leg pain) to explore the feasibility of a large, multicenter, pragmatic RCT. 
All statistical analysis will use a two-tailed test as a principle, and significance level will be set at $5 \%$. In addition, last observation carried forward (LOCF) will be conducted to handle missing data as it is currently widely used in clinical trials.

\section{Economic evaluation}

Economic evaluation will be performed to assess costeffectiveness of the three groups. The primary economic endpoint will be cost per quality-adjusted life year (QALY) gained, and secondary economic endpoints will cover such effectiveness parameters as cost per NRS. The primary analysis period will be 14 weeks and, if further estimation is needed, secondary analysis using regression-model or decision-modelling analysis to extrapolate cost and effect beyond the follow-up period will be performed. Treatment costs related to the clinical trial will be calculated by multiplying number of treatment sessions with unit cost, and unit costs will use national health insurance and medical institution cost data. Estimates of quality of life for QALYs will use the EQ-5D as the main assessment variable in the area under the curve method [34]. In the case that total time horizon is at least 12 months, the unit cost will be standardized using the Korean monetary unit (won) value as of 2013 and applying a discount rate of 5\% in accordance with Korean HIRA economic evaluation guidelines. The analysis perspective of this study will be social and, in baseline analysis, representative values (e.g., average) of study parameters will be used. All available distribution and representative values of parameter estimates will be applied in probabilistic sensitivity analysis.

\section{Data collection and management}

Paper CRFs will be used and a standard operating procedure (SOP) for CRF entry will be prepared. Investigators and the contract research organization (CRO) at the lead study site (Pusan National University Korean Medicine Hospital) will educate outcome assessors and investigators at each site regarding CRF entry, electronic data conversion, and SOP. Double data entry will be allowed in CRF outcome measurement data, with data entered once at each study site, and once at the lead organization and $\mathrm{CRO}$, respectively. After confirming whether electronic data conversion using double entry is accurate, data access will be blocked to all researchers except statisticians.

\section{Adverse events}

Physicians will monitor and record any unexpected or unintended patient reaction to Chuna, usual care, or its combination at each visit. Adverse events (AEs) associated with Chuna will include, but not be limited to, AEs anticipated from previous reports of manual therapy and will stay open to all possibilities taking into consideration other potential, unknown AEs. Physicians will rate the causal relationship between each treatment method and $\mathrm{AE}$ occurrence on a 6 -point scale $(1=$ definitely related; $2=$ probably related; 3 $=$ possibly related; $4=$ probably not related; $5=$ definitely not related; and $6=$ unknown), and all AEs will be categorized into three levels using the Spilker classification (mild $(1)=$ not needing additional intervention, nor significantly inhibiting to the normal lifestyle (function) of the participant; moderate $(2)=$ significantly inhibiting to the normal lifestyle (function) of the participant, and may need additional intervention, recovering afterwards; and severe (3) = severe $\mathrm{AE}$ requiring intensive intervention, and leaving sequelae). In the case of "serious adverse event (SAE)" during study duration, unblinding is permissible and the relevant Institutional Review Board (IRB) and main study site (Pusan National University Korean Medicine Hospital) will be informed and will decide whether the trial will continue or be terminated. In the event that a subject suffers direct injury associated with the clinical trial, the subject may receive appropriate medical action as seen fit by the subinvestigator, and damage compensation will follow prespecified clinical research insurance clauses. All study participants will be provided with an emergency contact number to the principal investigator or subinvestigator for any questions, medical issues or research-related diseases during the study and accordingly receive requested services.

\section{Data monitoring and safety monitoring}

This study is a pilot study with no interim analysis, and a data monitoring committee was not organized as no SAEs regarding Chuna or usual care not previously reported are expected to occur.

Safety assessment will mainly assess frequency of AEs, including all SAEs. AE reports will summarize the number and percentage of participants who experienced AEs, and will include categorized information on body region of AE manifestation. Other data (e.g., severity, causal relationship with treatment) will be included in safety monitoring reports.

An outside CRO will be entrusted with safety and data monitoring. CRO monitoring will consist of three sessions in the following order: initiative monitoring at first subject enrollment, one midterm monitoring during the clinical trial, and conclusive monitoring upon completion of clinical trial. Participant safety data and CRF and source documents will be reviewed and crosschecked to confirm participant safety and data integrity.

\section{Stopping rules}

The trial will be stopped in the case that the principle investigator regards that there is unacceptable risk of SAE(s) within group(s). 


\section{Discussion}

Usual care was selected as the choice of comparator in this study for investigation of the effectiveness and safety of Chuna manual therapy. Various national and international clinical practice guidelines and systematic reviews were consulted in discussion of usual care contents for LBP. While current guidelines indicate the benefits of conservative treatment [5], the evidence for individual conservative treatment methods and opinions on what should constitute standard care of LBP are somewhat inconsistent [4-6]. Moreover, recent reports state that the effect of acetaminophen, which is recommended for first-line use in LBP guidelines [4-6], is unclear [35]. Therefore, in this study we opted to use the most frequently used LBP treatments in Korea as opposed to an evidence-based selection. Korea has a unitary government-run national health insurance system which aims to cover the entire South Korean population and all medical institutions. This insurance system reimburses HIRA-approved medical costs for the greater majority of diseases including LBP, and the HIRA database contains extensive information on medication, medical services, and diagnosis [17]. For this study, we extracted the most frequently used treatment types in patients with a main diagnosis code of LBP from the 2011 HIRA national patients' sample (NPS) data, and provided this list to usual care providers for reference in patient treatment in an effort to secure validity in usual care treatment selection.

While the updated Cochrane review on spinal manipulative therapies for chronic LBP covers many study designs similar to the current study comparing spinal manipulation with other treatments, such as usual care, or as an add-on treatment to other treatments, such as usual care alone, evidence for the effect of spinal manipulative therapy is not conclusive [36]. However, as Chuna manual therapy and the spinal manipulative therapies included in the Cochrane review hold distinct differences, clinical effects may also be disparate. Meanwhile, a 2013 systematic review of RCTs on Chuna published in the Korean Chuna literature states that the two RCTs related to LBP both support a significant effect of Chuna, but that they are limited by qualitative issues and small sample size [37]. Therefore, it can be inferred from these evaluations that further rigorous clinical studies on Chuna, a Korean spinal manipulative therapy, are in order.

The aim of this pCRN study is to examine the comparative clinical effectiveness of Chuna, a Korean spinal manual therapy, through comparison of Chuna manual therapy and usual care treatments most frequently used in Korea, and investigate whether the combined treatment of Chuna manual therapy and usual care, which is reflective of, and more frequently encountered, than singular treatment of Chuna or usual care in real-world clinical practice, is more effective than single treatment. While this pilot study may fail to reach a definite conclusion due to current sample size, it will still hold relevance as one of the first well-designed, rigorous RCTs on Chuna manual therapy for nonacute LBP patients, and provide preliminary effectiveness and safety monitoring results for future large-scale, multicenter RCTs.

\section{Trial status}

Participant recruitment is anticipated to be from March to August 2016, and study completion to be reported in December 2016. The full-scale, multicenter RCT (CRN study) is expected to be initiated in 2017 after analysis of pCRN data.

\section{Additional files}

Additional file 1. SPIRIT (Standard Protocol Items: Recommendations fo Interventional Trials) 2013 Checklist. Description of data: populated SPIRIT (Standard Protocol Items: Recommendations for Interventional Trials) 2013 Checklist: recommended items to address in a clinical trial protocol and related documents. (DOC $123 \mathrm{~kb}$ )

Additional file 2. Trial committee organization and contributions and role. Description of data: trial committee organization and contributions and role in accordance with SPIRIT (Standard Protocol Items:

Recommendations for Interventional Trials) 2013 Checklist. (DOCX 29 kb)

Additional file 3. Chuna manual therapy technique application method. Description of data: technical details of Chuna manual therapy with photos. (DOCX $2822 \mathrm{~kb}$ )

\section{Abbreviations}

AE: Adverse event; ANCOVA: Analysis of covariance; ANOVA: Analysis of variance; CMT: Chuna manual therapy; CRF: Case Report Form; CRIS: Clinical Research Information Service; CRO: Contract research organization; EQ-5D: EuroQol-5 dimensions; HIRA: Health Insurance Review and Assessment; HUI-III: Health Utility Index III; IRB: Institutional Review Board; KMD: Korean medicine doctor; LBP: Low back pain; LOCF: Last observation carried forward; NPS: National patients' sample; NRS: Numeric Rating Scale; ODI: Oswestry Disability Index; pCRN: Pilot Chuna Research Network; PGIC: Patient Global Impression of Change; QALY: Qualityadjusted life years; RCT: Randomized controlled trial; ROM: Range of movement; SAE: Serious adverse event; SD: Standard deviation; SMT: Spinal manipulative therapy; SOP: Standard operating procedure; SPIRIT: Standard Protocol Items: Recommendations for Interventional Trials; SR: Systematic review; UC: Usual care

\section{Acknowledgements}

Not applicable.

Protocol versions

pCRN study protocol (original): 29 September 2015:

pCRN study protocol (amendment 01), 21 November 2015; pCRN study protocol (amendment 02), 26 November 2015

Other significant protocol revisions and amendments made after protocol publication will be notified through regular updates on the trial registration site (Clinical Research Information Service (CRIS)).

Trial committee organization and contributions and role See Additional file 2 .

\section{Funding}

This study was supported by the Traditional Korean Medicine R\&D program funded by the Ministry of Health and Welfare through the Korea Health Industry Development Institute (KHIDI) (Grant number: HI15C0103). The study design, execution, analysis, data interpretation and results publication will be performed independently of this funding source. 


\section{Availability of data and materials}

Not applicable.

\section{Authors' contributions}

B-CS conceived of, and will manage and oversee, this study as the primary investigator. $\mathrm{I}-\mathrm{HH}, \mathrm{E}-\mathrm{HH}, \mathrm{M}-\mathrm{rK}, \mathrm{J}-\mathrm{HC}, \mathrm{J}-\mathrm{YJ}, \mathrm{K}-\mathrm{WK}, \mathrm{KN}, \mathrm{MhL}$, and K-HH devised the original study design as experts in rehabilitation and clinical research. NK contributed to economic evaluation design and J-HL contributed through important suggestions for study execution and critically revised the protocol for significant intellectual content. All of the authors have read and approved the final manuscript.

\section{Competing interests}

The authors declare that they have no competing interests.

\section{Consent for publication}

\section{Not applicable.}

\section{Ethics approval and consent to participate}

This study has obtained relevant IRB approval at the four sites intended to participate in recruitment (Pusan National University Korean Medicine Hospital Institutional Review Board (2016002), Kyung Hee University Korean Medicine Hospital at Gangdong Institutional Review Board (KHNMC OH 2016-01-009), Jaseng Hospital of Korean Medicine Institutional Review Board (KNJSIRB2016-021), and Mokhuri Neck and Back Hospital Institutional Review Board (MHNBH-IRB-08)), and all clinical investigators involved in the study will receive education on the predetermined protocol, SOP, Helsinki Declaration, and Korean Good Clinical Practice Guidelines for the protection of study participants.

Written informed consent regarding trial participation will be obtained from all study participants. All material containing personal information of subjects will be rigorously managed under IRB governance, and personal information will be protected and kept confidential. All data collected from participants giving written informed consent to participate in this trial will be processed anonymously, and in the case that data is provided to other institutions for research means, it will be in the form of random codes excluding personal information. If the research objectives in use of data diverge from the current study, separate IRB approval will be required. Also, for the imperative use of personal information for research means, such as account copies for transportation fee transfer, the participant will be informed and a separate informed consent regarding personal information collection will be obtained.

\section{Author details}

'Spine and Joint Center, Pusan National University Korean Medicine Hospital, Yangsan, Republic of Korea. 'Department of Korean Rehabilitation Medicine, School of Korean Medicine, Pusan National University, Yangsan, Republic of Korea. ${ }^{3}$ Jaseng Spine and Joint Research Institute, Jaseng Medical Foundation, 858 Eonju-ro, Gangnam-gu, Seoul 135-896, Republic of Korea. ${ }^{4}$ Department of Korean Rehabilitation Medicine, Kyung Hee University, Seoul, Republic of Korea. ${ }^{5}$ Clinical Research Division, Korea Institute of Oriental Medicine, Daejeon, Republic of Korea. ${ }^{6}$ Korean Medicine Life Science, University of Science and Technology (UST), Campus of Korea Institute of Oriental Medicine, Daejeon, Republic of Korea. ${ }^{7}$ Mokhuri Neck and Back Hospital, Seoul, Republic of Korea. ${ }^{8}$ Center for Comparative Effectiveness Research and Economic Evaluation in Korean Medicine, Pusan National University, Yangsan, Republic of Korea.

Received: 14 June 2016 Accepted: 14 December 2016 Published online: 17 January 2017

\section{References}

1. Lambeek LC, Bosmans JE, Van Royen BJ, Van Tulder MW, Van Mechelen W, Anema JR. Effect of integrated care for sick listed patients with chronic low back pain: economic evaluation alongside a randomised controlled trial. BMJ. 2010;341:C6414.

2. Shiri R, Karppinen J, Leino-Arjas P, Solovieva S, Varonen H, Kalso E, Ukkola O, Viikari-Juntura E. Cardiovascular and lifestyle risk factors in lumbar radicular pain or clinically defined sciatica: a systematic review. Eur Spine J. 2007;16: 2043-54.
3. Rubinstein SM, van Middelkoop M, Assendelft WJ, de Boer MR, van Tulder MW. Spinal manipulative therapy for chronic low-back pain: an update of a Cochrane review. Spine (Phila Pa 1976). 2011;36:E825-46.

4. Koes BW, van Tulder M, Lin CW, Macedo LG, McAuley J, Maher C. An updated overview of clinical guidelines for the management of non-specific low back pain in primary care. Eur Spine J. 2010;19:2075-94.

5. Chou R, Qaseem A, Snow V, Casey D, Cross Jr JT, Shekelle P, Owens DK, Clinical Efficacy Assessment Subcommittee of the American College of Physicians, American College of Physicians, American Pain Society Low Back Pain Guidelines Panel. Diagnosis and treatment of low back pain: a joint clinical practice guideline from the American College of Physicians and the American Pain Society. Ann Intern Med. 2007;147:478-91.

6. van Tulder M, Becker A, Bekkering T, Breen A, del Real MT, Hutchinson A, Koes B, Laerum E, Malmivaara A, COST B13 Working Group on Guidelines for the Management of Acute Low Back Pain in Primary Care. Chapter 3. European guidelines for the management of acute nonspecific low back pain in primary care. Eur Spine J. 2006;15 Suppl 2:S169-91.

7. Park JM, Shin SW, Park J. A comparative study on the concepts of the Chuna (推拿). The Journal of Korean Medical Classics. 2008;21:173-91.

8. Hwang M, Cho H, Lee H, Heo K, Hwang E, Shin M, Shin B. Research trends on Chuna treatment in Korean medicine - Focused on type of clinical trials, published year, academic journals and treatment technique for each used parts. The Journal of Korea Chuna Manual Medicine for Spine \& Nerves. 2013;8:49-61.

9. Schulz KF, Altman DG, Moher D, CONSORT Group. CONSORT statement: updated guidelines for reporting parallel group randomized trials. Ann Intern Med. 2010;2010(152):726-32.

10. Cho H, Lee H, Heo K, Hwang E, Shin B, Shin M. Randomized controlled clinical trials in the Journal of Korean Society of Chuna Manual Medicine for Spine and Nerves: a quantitative and qualitative analysis. The Journal of Korea Chuna Manual Medicine for Spine \& Nerves. 2012;7:15-23.

11. Cho JG, Kim NS, Do SR, Lee YH, Yoon GJ, Park JH, Jang DH, Chun JY, Kim HY, Lee NH, Yoo HS, Seo SW. 2011 National Survey on the use of Korean Medicine and Korean Herbal Medicine. Sejong: Korea Ministry of Health and Welfare, Korea Institute for Health and Social Affairs; 2012. 11-1352000000547-12:1-554.

12. Park J, Kwon SM. Determinants of the utilization of oriental medical services by the elderly. J Korean Oriental Med. 2011;32:97-108.

13. Ko Y, Lee J, Hwang E, Heo K, Yun J, Park T, Kong J, Sul J, Jung T, Kim K, Yang $H$, Shin B. A study to provide of health insurance for Chuna manual therapy. J Korea Chuna Manual Med Spine Nerves. 2012;7:1-14.

14. Kim B, Hwang E, Heo K, Hwang M, Heo I, Song Y, Lee J, Ko Y, Park T, Cho J, Lee E, Mun S, Ha I, Lee M, Shin B. The Survey on the Standardization of Chuna Manual Technique for Operating RCT of Non-acute Low Back Pain. J Korea Chuna Manual Med Spine Nerves. 2015;10:13-25.

15. Shin YS, Shin JS, Lee J, Lee YJ, Kim MR, Ahn YJ, Park KB, Shin BC, Lee MS, Kim JH, Cho JH, Ha IH. A survey among Korea Medicine doctors (KMDs) in Korea on patterns of integrative Korean Medicine practice for lumbar intervertebral disc displacement: preliminary research for clinical practice guidelines. BMC Complement Altern Med. 2015;15:432.

16. Park JJ, Shin J, Choi Y, Youn Y, Lee S, Kwon SR, Lee H, Kang MH, Ha IH, Shin I. Integrative package for low back pain with leg pain in Korea: a prospective cohort study. Complement Ther Med. 2010;18:78-86.

17. Health Insurance Review and Assessment Service. http://www.hira.or.kr/. Accessed 5 Jan 2017.

18. Farrar JT, Young Jr JP, LaMoreaux L, Werth JL, Poole RM. Clinical importance of changes in chronic pain intensity measured on an 11-point numerical pain rating scale. Pain. 2001;94:149-58.

19. Turk DC, Rudy TE, Sorkin BA. Neglected topics in chronic pain treatment outcome studies: determination of success. Pain. 1993;53:3-16.

20. Fairbank JC, Pynsent PB. The Oswestry Disability Index. Spine (Phila Pa 1976). 2000;25:2940-52. discussion 2952.

21. Jeon $\mathrm{CH}$, Kim DJ, Kim SK, Kim DJ, Lee HM, Park HJ. Validation in the crosscultural adaptation of the Korean version of the Oswestry Disability Index. J Korean Med Sci. 2006;21:1092-7.

22. Guy W. National Institute of Mental Health. Psychopharmacology Research Branch, Early Clinical Drug Evaluation Program: ECDEU assessment manual for psychopharmacology. Rockville: U.S. Dept. of Health, Education, and Welfare, Public Health Service, Alcohol, Drug Abuse, and Mental Health Administration, National Institute of Mental Health, Psychopharmacology Research Branch, Division of Extramural Research Programs; 1976. 
23. Group EQ. EuroQol-a new facility for the measurement of health-related quality of life. Health Policy. 1990;16:199-208.

24. Nam HS, Kim KY, Kwon SS, Koh KW, Poul K. EQ-5D Korean valuation study using time trade-off method. Cheongwon: Korea Centers for Disease Control and Prevention; 2007.

25. Kopec JA, Willison KD. A comparative review of four preference-weighted measures of health-related quality of life. J Clin Epidemiol. 2003;56:317-25.

26. Saur PM, Ensink FB, Frese K, Seeger D, Hildebrandt J. Lumbar range of motion: reliability and validity of the inclinometer technique in the clinical measurement of trunk flexibility. Spine (Phila Pa 1976). 1996;21:1332-8.

27. Hart DL, Werneke MW. Re: Pengel LHM, Refshauge KM, Maher CG. Responsiveness of pain, disability, and physical impairment outcomes in patients with low back pain. Spine. 2004;29:879-83. Spine (Phila Pa 1976). 2004;29:2475-6.

28. Gold MR, Patrick DL, Torrance GW, Fryback DG, Hadorn DC, Kamlet MS, Daniels N, Weinstein MC. United States Public Health Service Panel on Costeffectiveness in Health and Medicine. Identifying and valuing outcomes. In: Gold MR, Siegel JE, Russell LB, Weinstein MC, editors. Cost-effectiveness in health and medicine. New York: Oxford University Press; 1996. p. 82-134.

29. Berger ML. International Society for Pharmacoeconomics and Outcomes Research. Health care cost. Quality, and outcomes: ISPOR book of terms. Lawrenceville: International Society for Pharmacoeconomics and Outcomes Research (ISPOR); 2003.

30. Rascati KL. Essentials of pharmacoeconomics. 1st ed. Philadelphia: Wolters Kluwer/Lippincott Williams \& Wilkins; 2009.

31. Devilly GJ, Borkovec TD. Psychometric properties of the credibility/ expectancy questionnaire. J Behav Ther Exp Psychiatry. 2000;31:73-86.

32. Shin JS, Ha IH, Lee J, Choi Y, Kim MR, Park BY, Shin BC, Lee MS. Effects of motion style acupuncture treatment in acute low back pain patients with severe disability: a multicenter, randomized, controlled, comparative effectiveness trial. Pain. 2013;154:1030-7.

33. Yeung CK, Leung MC, Chow DH. The use of electro-acupuncture in conjunction with exercise for the treatment of chronic low-back pain. J Altern Complement Med. 2003;9:479-90.

34. Brazier J, Ratcliffe J, Salomon JA, Tsuchiya A. Measuring and valuing health benefits for economic evaluation. Oxford: Oxford University Press; 2007.

35. Machado GC, Maher CG, Ferreira PH, Pinheiro MB, Lin CW, Day RO, McLachlan AJ, Ferreira ML. Efficacy and safety of paracetamol for spinal pain and osteoarthritis: systematic review and meta-analysis of randomised placebo controlled trials. BMJ. 2015;350:h1225.

36. Rubinstein SM, Terwee CB, Assendelft WJ, de Boer MR, van Tulder MW. Spinal manipulative therapy for acute low-back pain. Cochrane Database Syst Rev. 2012;9:CD008880.

37. Moon TW, Choi TY, Park TY, Lee MS. Chuna therapy for musculoskeletal pain: a systematic review of randomized clinical trials in Korean literature. Chin J Integr Med. 2013;19:228-32.

38. Korean Society of Chuna Manual Medicine for Spine \& Nerves. Chuna medicine. Seoul: Korean Society of Chuna Manual Medicine for Spine \& Nerves; 2014.

39. The Society of Korean Rehabilitation. Oriental Rehabilitation Medicine. 3rd ed. Seoul: Koonja; 2011.

40. DeStefano LA. Greenman's principles of manual medicine. 5th ed. Philadelphia: Lippincott Williams \& Wilkins; 2016.

41. Bergmann TF, Peterson DH. Chiropractic technique: principles and procedures. 3rd ed. St. Louis: Mosby; 2010.

\section{Submit your next manuscript to BioMed Central and we will help you at every step:}

- We accept pre-submission inquiries

- Our selector tool helps you to find the most relevant journal

- We provide round the clock customer support

- Convenient online submission

- Thorough peer review

- Inclusion in PubMed and all major indexing services

- Maximum visibility for your research

Submit your manuscript at www.biomedcentral.com/submit 